\title{
Improving the methodology for observing deformations of buildings and structures
}

\author{
Olga Tsareva ${ }^{1, *}$ and Fedor Portnov ${ }^{2}$ \\ ${ }^{1}$ Peter the Great St.Petersburg Polytechnic University, 29, Politechnicheskaya Str., St. Petersburg, \\ 195251, Russia \\ ${ }^{2}$ Moscow State University of Civil Engineering, Yaroslavskoe shosse, 26, Moscow, 129337, Russia
}

\begin{abstract}
The article presents the observation and evaluation method for deformations based on the use of spatial polar intersection, which is implemented by tacheometers. In the classic version, observations are carried out from fixed stations to determine the coordinates of the deformation marks, while the instrument is centered, leveled and oriented. The proposed methodology is based on two technologies for performing work: from fixed points and from free stations. When using fixed points in the first version, the total station is centered, leveled and oriented to a remote target that is the same for all stations. In the second variant, in the absence of a common remote target, measurements are made of the magnetic azimuths of the directions used to orient the instrument. In both cases, it is not necessary to have change points between stations. The option of transit traverse is possible. When observing from free stations without using change point and reference marks, it is proposed to proceed to invariant values for estimating deformations. Such invariants, in particular, are the distances between marks. The results can be used to observe the deformations of various buildings and structures.
\end{abstract}

\section{Introduction}

It is important to obtain displacement vectors and the location of the object points to which these vectors belong (for example, the coordinates of the deformation marks) during observing deformations for analyzing the stability of an object. Required accuracy of specified parameters is different. A module and a direction characterize a vector in space. The regulatory documents define the required accuracy of the module's assessment. The acceptable error of their determination, as a rule, is the a few millimeters, and for earthworks is $10-15 \mathrm{~mm}$ [1]. There are no tolerances in the direction, which means that an error in estimating the vector direction even by several degrees does not matter in information analyze. The accuracy of positioning the application point of the vector is also not normalized, and the indication of its coordinates with errors in centimeters or even decimeters for conclusions about the state of the object is not critical. Taking into account the above in the development process of observation project can significantly influence the technology of field measurements.

*Corresponding author: tsareva_os@spbstu.ru 
In articles [2-13] consider some methods for deformation observing and as conclusion is taken that the polar method of determining coordinates (spatial polar intersection) is best. There some options for its implementation.

\section{Methods}

Suppose that during object observation we use a spatial polar intersection from electronic total interconnected stations (Fig. 1). In this case, there are two possible technologies for performing work: measurements from fixed points, measurements from free stations.

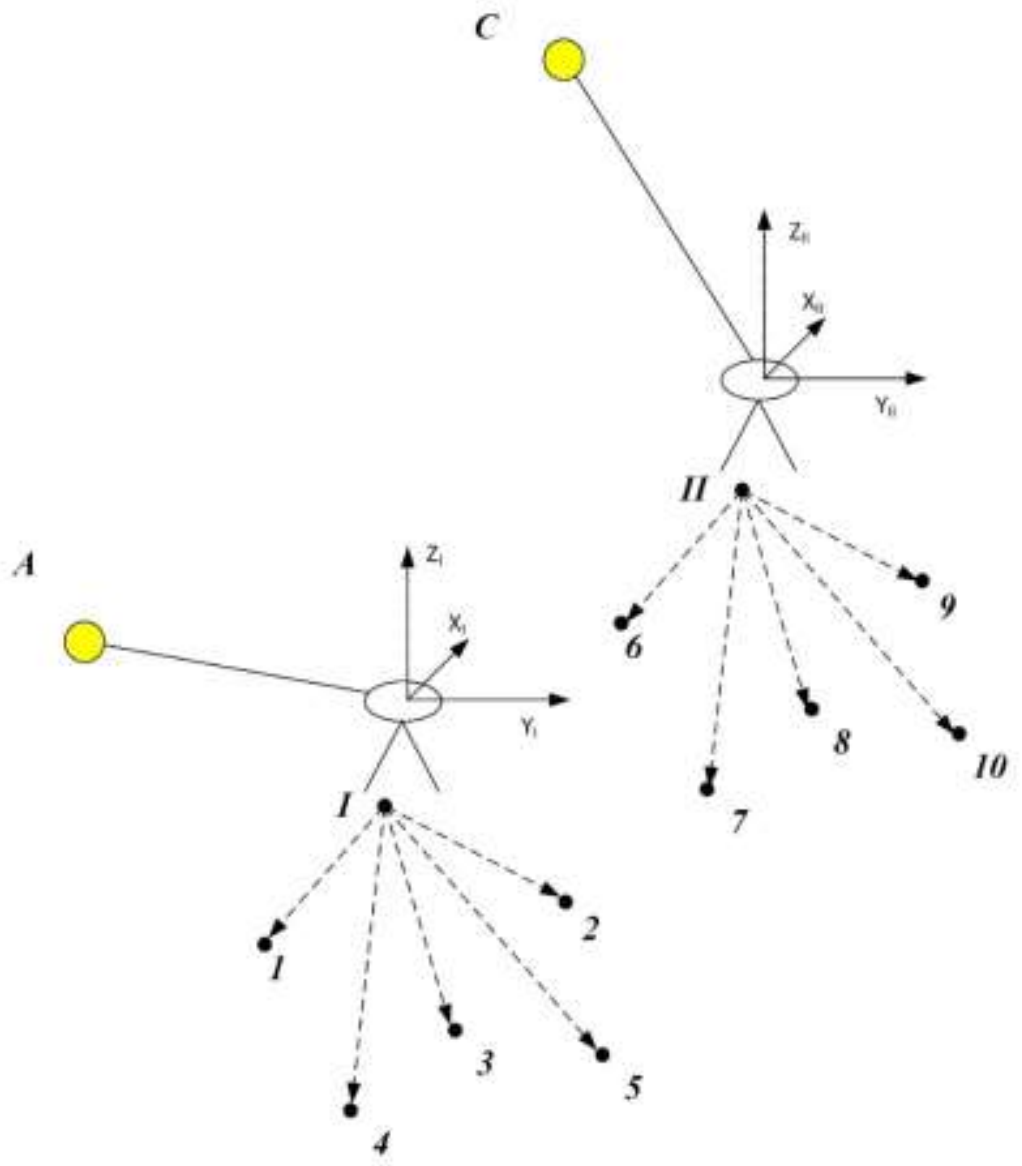

Fig. 1. Spatial polar intersection: I, II - tachometer station points, 1-10 - deformation marks, A, C reference marks.

The first one implies fixing each station with a center and installing reference marks to orient the total station. In this case, the process of bringing the device to the working position in any measurement cycle provides for three obvious procedures (centering, leveling and orientation). The components of the displacement vectors $\Delta X, \Delta Y, \Delta Z$ in this case are equal to the difference of coordinates obtained from the subsequent and previous cycles of observations, since the measurements of the first and subsequent cycles are made in the same coordinate system. But at different stations different coordinate system and the analysis of 
the whole object as a whole will be performed if the vectors are defined in a single coordinate system.

In the classic version, common reference marks (fig.2) or change points (fig. 3) perform the transformation of the point coordinates obtained from different stations to a single coordinate system. This is a classic method of observation, which requires the presence of reference marks or change points between stations in the right quantity.

In particular, figure 2 shows reference marks $\mathrm{A}, \mathrm{B}, \mathrm{C}$ for two station points.

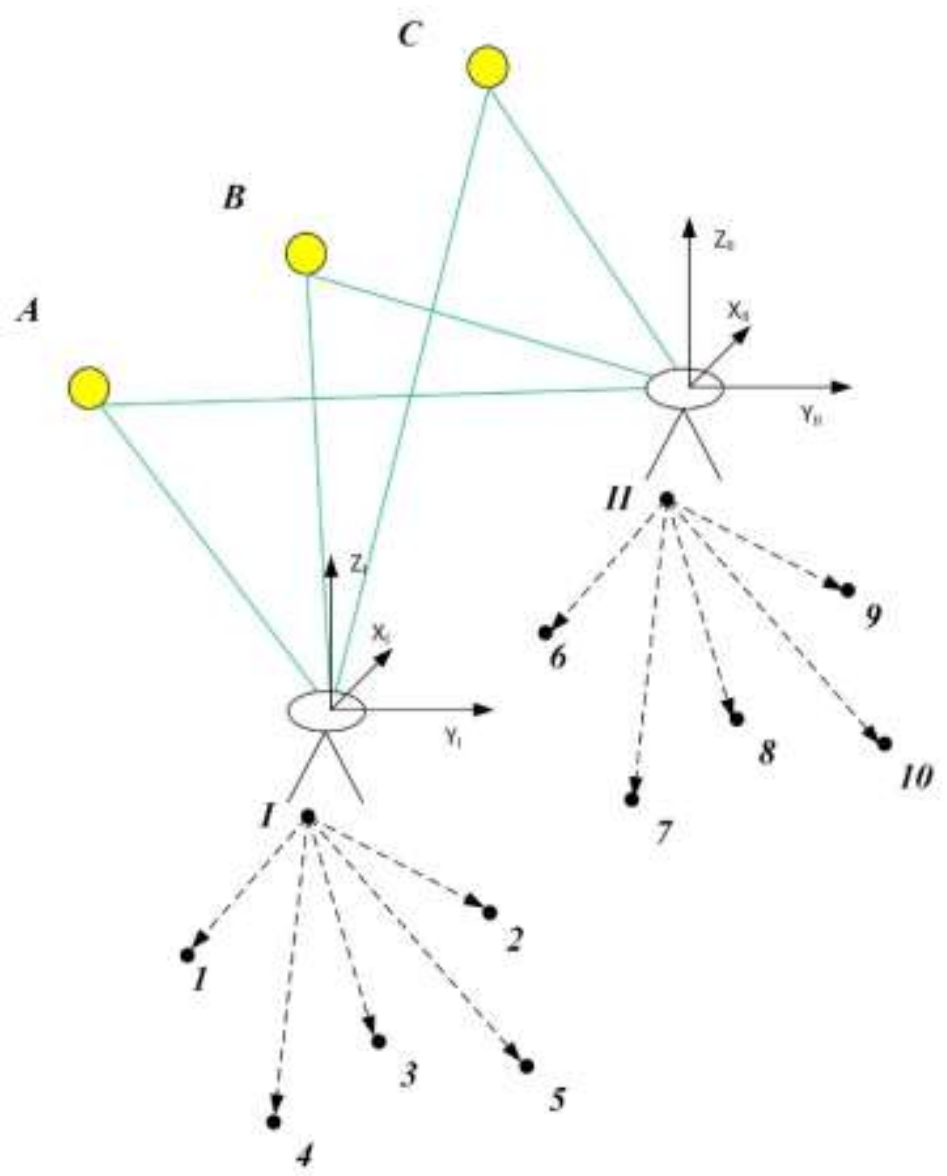

Fig. 2. Spatial polar intersection: I, II - tachometer station points, 1-10 - deformation marks, A, B, C reference marks.

In case there is no visibility to the reference marks from each station, the transformation of the mark coordinates into a single coordinate system is performed by change points (Figure 3): 


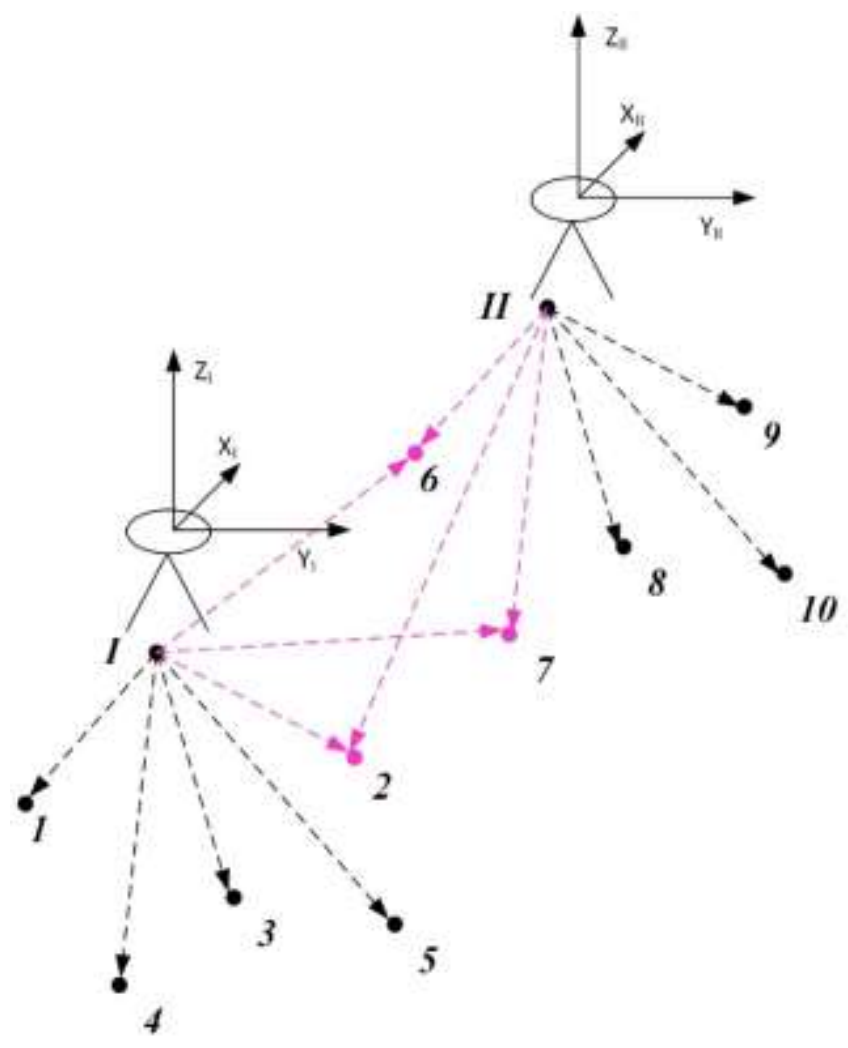

Fig. 3. Spatial polar intersection using change points $2,6,7$ between stations.

In the absence of reference marks or change points between stations in sufficient quantity, the following options are possible to solve the problem of determining displacement vectors in a single coordinate system:

The first option involves the use of the coordinates of the deformation marks taken from the observation project, drawn up according to the drawings of the object (its digital models, built in a single coordinate system). In this case, the requirement that the vectors be evaluated in a single coordinate system for the whole object is eliminated, since the points of their application are known, and for analyzing the state of the object, the mutual parallelism of the coordinate axes is sufficient. In other words, displacement vectors will be defined in identically oriented coordinate systems. When assessing the direction of the displacement vector, it is taken into account that, provided the device is level $Z_{I}$ and $Z_{I}^{\prime}$ mutually parallel. Only axis $X$ and $Y$ is turned at some angle. Therefore, it is possible to use for orientation of the instrument the same target for all observation cycles, but the target at a distance that is sufficiently far away (that is, the distance that can be taken as the coordinate axes $X$ and $Y$ at all stations mutually parallel). Then the displacement vector of all the marks will be obtained in the equally oriented coordinate system and defined as the difference between the coordinates of the initial and the current observation cycles. In fig. 4 green color represents a reference mark (remote building whose spire is used to orient the tacheometer total station at each station). 


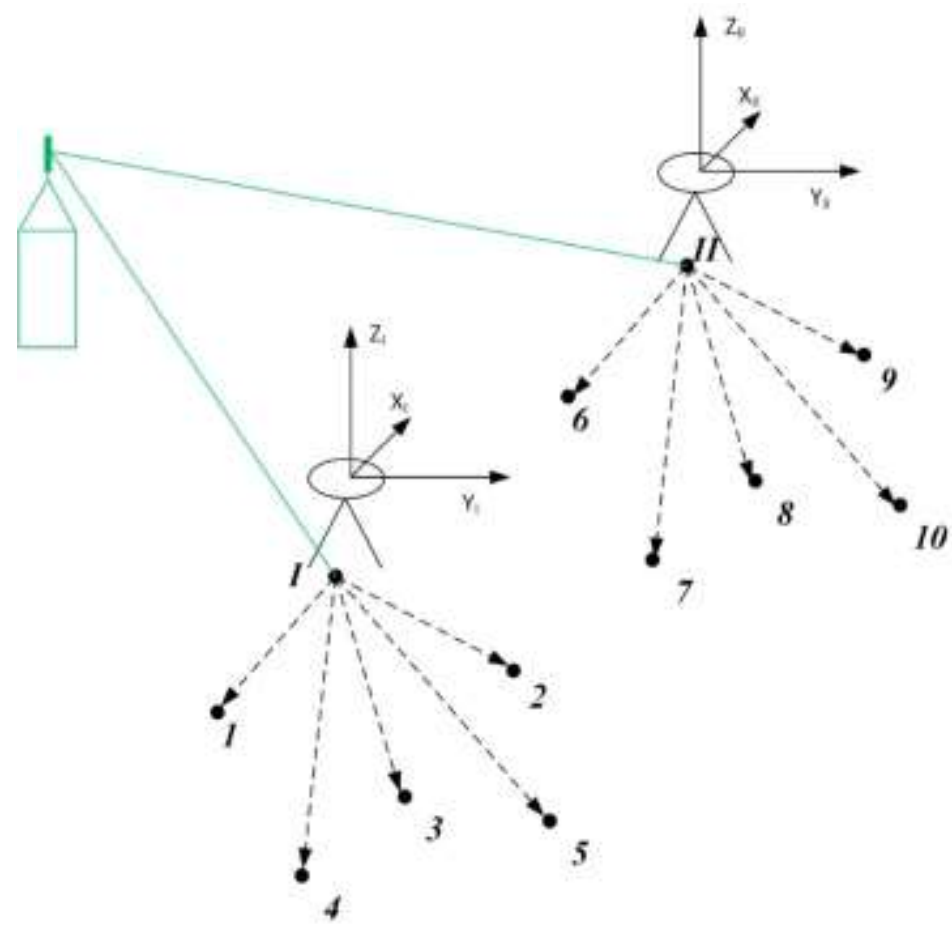

Fig. 4. Using a remote sign to orient the tacheometer.

In the general case you have to rotate the coordinate systems in the horizontal plane. But you need to know at what angle. It can be estimated, for example, by the difference of the directional angles (magnetic azimuths) of the orientation directions (polar axes). In this case, the program of field work should include either the location of the so-called directional movement, or the measurement of magnetic azimuths of reference directions.

In the transit traverse, which can also be laid by a theodolite of technical accuracy, all horizontal angles are measured, between the sides of the stroke, as well as angles to the orienting directions, but the lengths of its sides as unnecessary are not measured.

A variant of such transit traverse is presented in Figure 5. It contains black circles showing the stations from which object points are measured (deformation marks), yellow circles are reference marks for the orientation of tacheometers, a white circle is an auxiliary point of travel, and measured horizontal angles are shown in arcs.

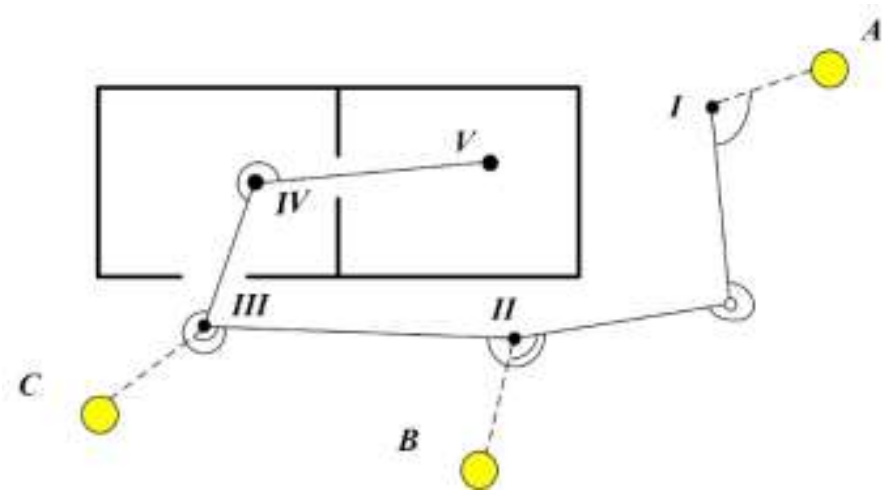

Fig. 5. Transit traverse: I - V - tachometer station points, A, B, C - reference marks. 
At stations IV and V there are no signs to orient the tacheometers, therefore, for these purposes it is necessary to use adjacent observation stations.

It is clear that the directional angles can be calculated both from the known side (if it is in the scheme), as well as from some conditional value. From Figure 5 it can be seen that in this way external stations are simply connected to stations located inside the premises.

In accordance with the third option, it is supposed to determine the coordinates by orienting the device at each station to any closely located target (for example, reference marks $B$ or $C$ ) and measurement of magnetic azimuths of directions used to orient the instrument. Then turn the axes $X$ and $Y$ will be equal to the difference of the magnetic azimuth directions. In fig. 6 blue shows the north directions of the magnetic meridian at each station.

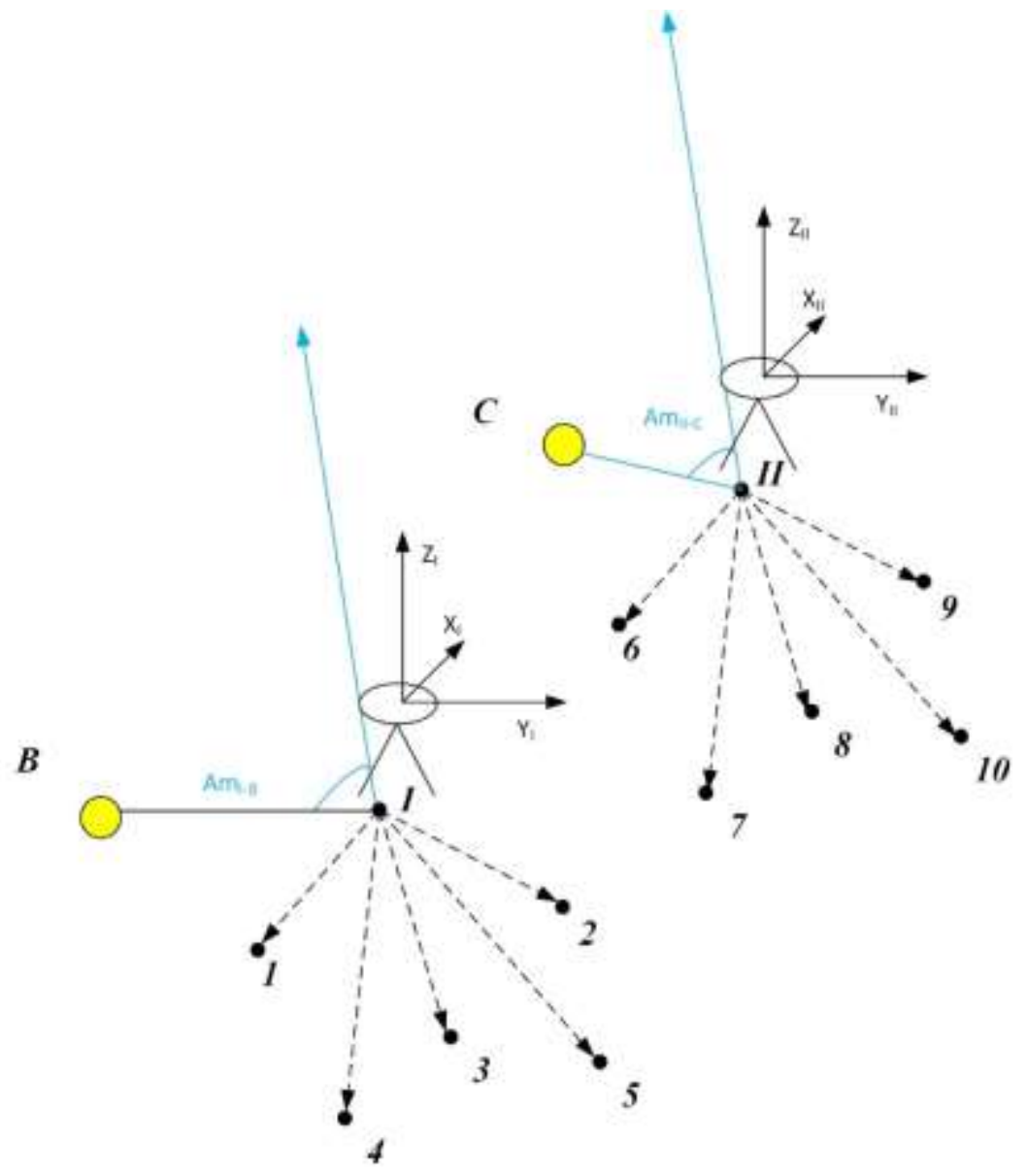

Fig. 6. Measurement of magnetic azimuths of orientation directions.

When using free stations (Fig. 7), in order to have the coordinates of marks in the previous and subsequent cycles of observations in one system, it is required to perform their conversion by change points. Only then can the components of the displacement vectors be estimated from the coordinates differences. In the case of observations from free stations, there are no change points. Then it is not possible to determine the displacement vectors, since the coordinates of the marks are obtained in different coordinate systems that are not related to each other. In this regard, an idea arises to use invariant parameters with respect to 
the coordinate system transformation when assessing the stability of buildings and structures. Such invariants, in particular, are the distances between the deformation marks.

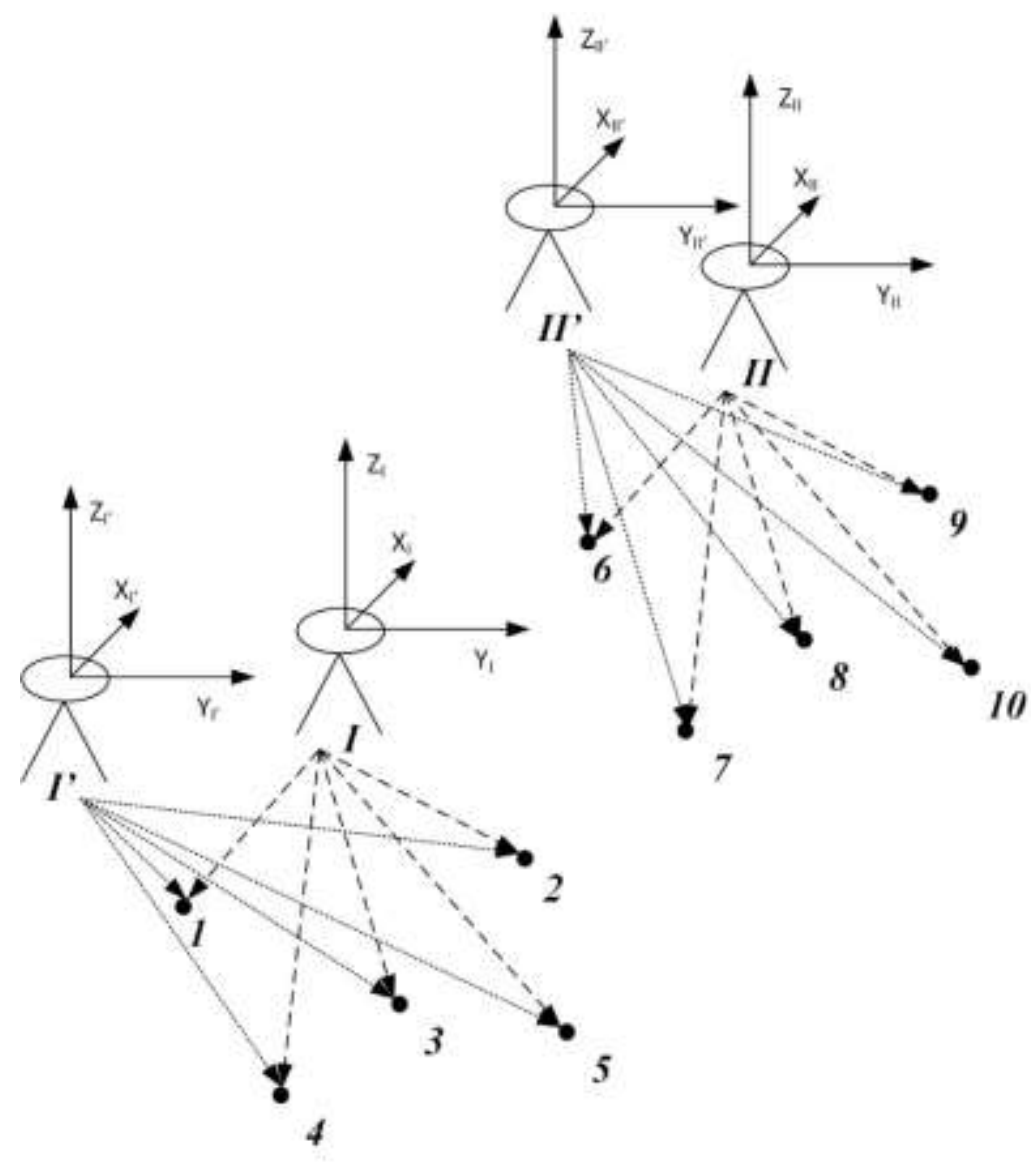

Fig. 7. Scheme for determining the coordinates of marks from a free station: $I, I^{\prime} \quad I I, I I^{\prime}$ free stations, 1-10 - deformation marks.

Distances can be calculated by coordinates, or directly measured. After that, it is possible to determine the vector of displacement of marks in a single coordinate system for each station. To do this, perform network equalization as free using the least squares method [14].

\section{Results and discussion}

Thus, with different methods of measurements, the displacement vectors of all the marks were obtained in a single object coordinate system or in an equally oriented coordinate system. Next, the resulting displacement vectors are analyzed for the presence of deformations and the type of deformation is determined [15].

The studies made allow us to draw the following conclusion: taking into account the accuracy requirements for estimating the parameters of displacements of object elements (coordinates of application points of displacement vectors, their modules and directions) significantly affects the technology of measurements performed while studying deformation processes, and allows them to be significantly simplified. 


\section{Conclusions}

A new approach to measurements is presented:

1. When observing from fixed points, the following options are possible:

a) use only one remote reference mark during observing instead of three (removed at a sufficient distance so that the axes of coordinates and at all stations can be considered mutually parallel). When estimating the direction of the displacement vector, it is taken into account that, provided that the device is level, the axes and are mutually parallel.

b) measurement of magnetic azimuth directions. Then, to obtain the vectors of marks offsets in an equally oriented coordinate system, it is necessary to rotate the coordinate axes by an angle equal to the difference of the magnetic azimuths of the directions.

c) transit traverse, in which all horizontal angles between the sides of the stroke are measured, as well as angles to the orientations.

In the proposed variants, there is no need for connect points between the station points, especially if it is impossible, for one reason or another, to make observations on them. Then the vector of the displacements of marks get in the equally oriented coordinate system.

2. When observing from free stations: to obtain displacement vectors in a single coordinate system of the object, they switch to invariant parameters - distances between deformation marks.

3. The developed method of observation can be used in observing the deformations of various buildings and structures for rapid assessment of the stability of the object.

\section{References}

1. Russian Federation Standard GOST 24846 - 2012

2. I. Puente, R. Lindenbergh, A. Van Natijne, R. Esposito, R. Schipper, ISPRS Archives 42(2), 923-929 (2018)

3. L.F. Zhou, P.F. Ma, Y. Xia, C.H. Xie, ISPRS Archives 42(3), 2535-2539 (2018)

4. M. Wróblewska, E3S Web of Conferences 36 (2018)

5. M. Talich, Y. Bao, W. Guo, G. Wang, W. Gan, M. Zhang, J.S. Shen, E. Kuzina, V. Rimshin, ISPRS Archives 42(3W4), 499-504 (2018)

6. Y. Bao, W. Guo, G. Wang, W. Gan, M. Zhang, J.S. Shen, Journal of Surveying Engineering 144(1) (2018)

7. E. Kuzina, V. Rimshin, Advances in Intelligent Systems and Computing 692, 410$416(2018)$

8. ̌. Rákay, S. Labant, K. Bartoš, Geodesy and Cartography 44 (1), 14-21 (2018)

9. M.Talich, IOP Conference Series: Earth and Environmental Science 95(3) (2017)

10. L. Wang, L. Yan, H. Hu, ICCSS 2017 - 2017 International Conference on Information, Cybernetics, and Computational Social Systems, 611-613 (2017)

11. H.-G. Maas, Photogrammetric Engineering and Remote Sensing 68(9), 933-940 (2002)

12. S. Erol, B. Erol, T. Ayan, ISPRS Archives 35, 622-627 (2004)

13. Z.-Y. Hou, D.-J. Yu, Wutan Huatan Jisuan Jishu, 26(3), 256-259 (2004)

14. M. Pieraccini, G. Luzi, D. Mecatti, M. Fratini, F. Parrini, G. Pinelli, C. Atzeni, PIERS 2004 - Progress in Electromagnetics Research Symposium, Extended Papers Proceedings, 643-646 (2004)

15. S.V. Tyurin, Journal of Mining Institute 156, 193-197 (2014)

16. O.S. Tsareva, Earthquake engineering. Constructions safety 5, 29-37 (2018) 